\title{
SIMULATION-BASED SCHEDULING FOR PHOTO-RECONNAISSANCE SATELLITE
}

\author{
Qiming Ruan \\ Yuejin Tan \\ Renjie He \\ Yingwu Chen \\ School of Information System and Management, \\ National University of Defense Technology, \\ Changsha, Hunan Province, CHINA
}

\begin{abstract}
A simulation-based scheduling mechanism for photoreconnaissance satellite is presented in this paper. The satellite scheduling problem belongs to a class of singlemachine scheduling problems with time window constraint It is NP-hard in computational complexity. Based on simulation platform, a mixed integer programming model is used to formulate the problem and an advanced tabu algorithm is adopted to solve the MIP. Numerical results demonstrate that this approach is efficient in the scheduling problems.
\end{abstract}

\section{INTRODUCTION}

Photo-reconnaissance satellite is operated from earth to meet military image requests of certain zones. Generally, there are more image requests for satellite than that can be satisfied, the satellite scheduling is an oversubscription scheduling problem. Satellite just has limited windows of opportunity to image a given target area, its activity scheduling must meet the strict and demanding requirements. Solving the scheduling problem can make the maximum use of satellite.

The imaging request usually consists of spot targets and area targets. A spot target is a small circular or rectangle area, while an area target is a large irregular polygonal. Compared with spot target, area target is much larger. An area target requires several contiguous strips to be imaged, while a spot target can be covered by a single strip with steady posture. Although the study on satellite scheduling problems about spot target has attracted much attention for years, the study about area target is still needed to be further investigated.

The time window is a primary constraint needed to be met. In order to achieve repetitive observation under comparable light conditions, photo-reconnaissance satellite is usually launched into heliosynchronous orbit. Based on the characteristics of heliosynchronous orbit, a given target zone just have limited time windows of opportunity for being imaged.

To agile satellite whose sensor can point cross-track or along-track, it takes time to maneuver the satellite-based sensor from its previous position to the desired aspect angle for the new imaging operation. The maneuvering time, also called setup time, depends on the positions and postures of the two consecutive imaging operation. For a given imaging area, the choice of the position and posture for the satellite is not unique. There are preferences for the possible combination of positions and postures. Since the time windows are fixed, the maneuvering operation should consider the imaging time to ensure that there are enough time to image the selected target.

According to the above discussion, we conclude that the satellite imaging scheduling problem belongs to a class of single-machine scheduling problems featured by sequence-dependent setup effects and time window constraints, which is NP-hard in computational complexity.

To solve the satellite scheduling problem, this paper presents a scheduling mechanism based on simulation. The scheduling mechanism will be described in the following sections.

\section{SIMULATION-BASED SCHEDULING MECHANISM}

In the scheduling mechanism, simulation is the foundation of scheduling system, which fulfills the functions of preprocessing and validating. The simulation-based scheduling mechanism consists of four distinct phases: constructing scenario, processing data, scheduling and validating scheme (see the Figure 1). The roles of these phases are outlined in the following subsections. 


\subsection{Constructing Scenario}

Constructing Scenario is the stage of establishing a scenario in simulation module while the scheduling mission is arrived. The constructed scenario includes all information about targets, satellite, satellite-based sensor, groundstation, time period, photograph weight, light constraint and other constraints. Every scenario represents a scheduling mission, which can be kept in the archives.

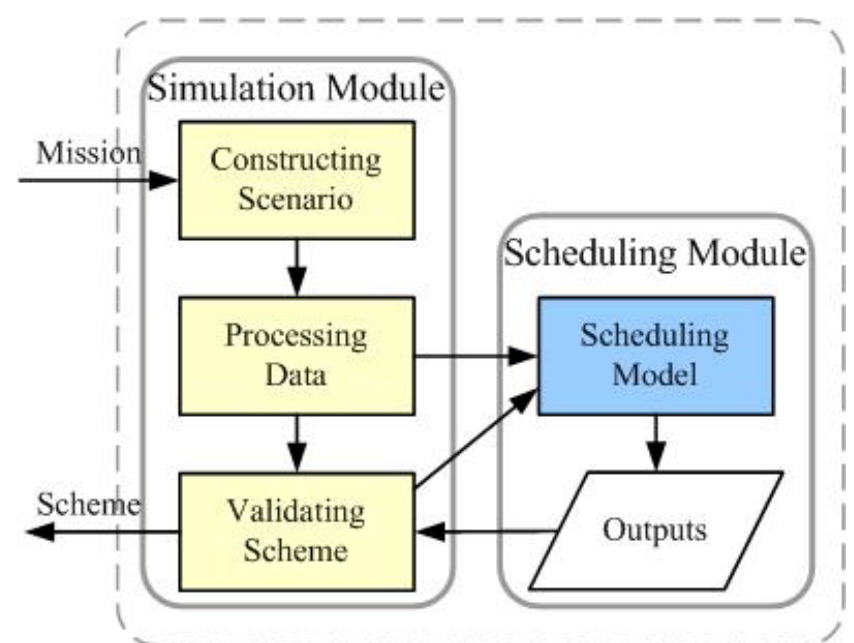

Figure 1: Simulation-based $\overline{\text { Scheduling }} \overline{\text { Mechanism }}$

\subsection{Processing Data}

At this stage, the simulation module will compute the time windows of imaging targets and then output data of time windows to scheduling module. Considering the characteristic of satellite-based sensor, a difference between spot target and area target is made. Let $\mathfrak{R}^{\prime}$ denote the set of spot targets and $\mathfrak{R}^{\prime \prime}$ be the set of area targets. $\mathfrak{R}$ represents the whole targets required to be imaged, where $\mathfrak{R}=\mathfrak{R}^{\prime} \cup \Re^{\prime \prime}$.

The time windows of spot target can be computed directly with the position. Compared with spot target, the computing procedure for area target is much more complicated. Simulation module will first compute the polynomial equation of satellite nadir based on the parameters of orbit, then divide area target into many segments based on the polynomial equation. The direction of every segment is parallel to the track of satellite. The size of segment has a reference to the designing characteristic of satellite-based sensor (see Figure 2). Let $\mathfrak{R}^{\prime \prime \prime}$ be the set of candidate pieces which are the divided result of area target in $\mathfrak{R}^{\prime \prime} . I$ denote the set of all candidate tasks, which will be outputted into scheduling module, then $I=\mathfrak{R}^{\prime} \cup \mathfrak{R}^{\prime \prime \prime}$.

For each imaging task $i \in I$, we use the simulation module to compute time windows with the constraint of scenario time period. Note $T W^{i}$ represents the set of can- didate time windows about task $i$, where $T W^{i}=\left\{T W_{1}^{i}, \cdots, T W_{N_{i}}^{i}\right\}, T W_{n}^{i}=\left[w s_{n}^{i}, w e_{n}^{i}\right], n \in\left\{1, \cdots, N_{i}\right\}$. $N_{i}$ is the number of time windows which correspond to target $i . w s_{n}^{i}$ and $w e_{n}^{i}$ are the time frames available for processing task $i$.

In this scheduling problem, we define every target $r(r \in \mathfrak{R})$ with the weight $W_{r}$, which is related to the priority and acreage of target. While an area target $r\left(r \in \mathfrak{R}^{\prime \prime}\right)$ is divided into $\mathrm{M}_{r}\left(\mathrm{M}_{r} \geq 2\right)$ pieces of segment, the weight of task $i\left(i \in \mathfrak{R}^{\prime \prime \prime}\right)$ which is derived from target $\mathrm{r}$ can be calculated with equation $W_{r} / \mathbf{M}_{r}$.

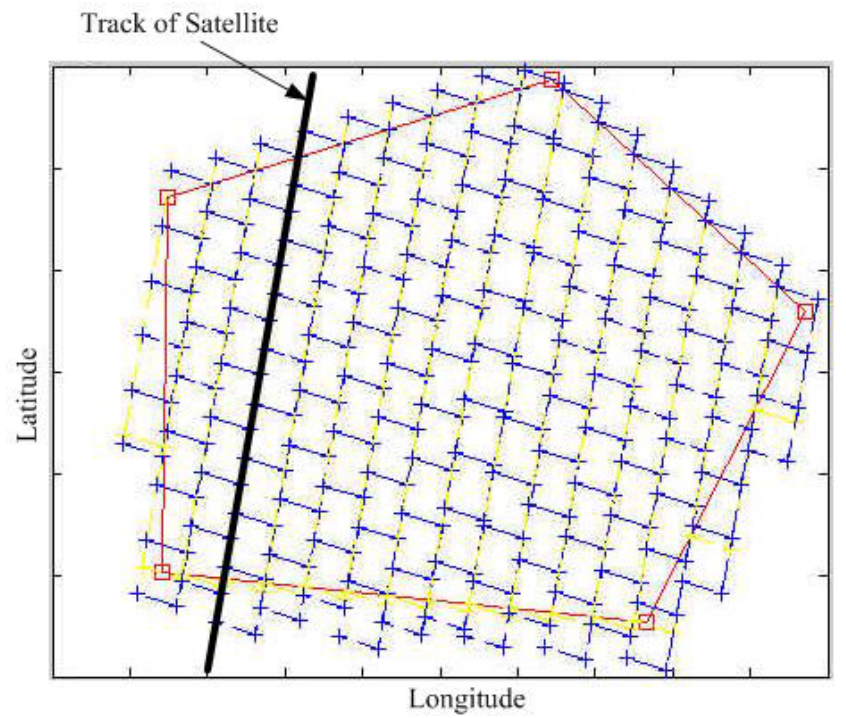

Figure 2: The cutting up of an area target into segments of constant size. The area target is represented by a red line, and the resulting images by blue lines

\subsection{Scheduling}

We construct a mixed integer programming (MIP) model to formulate the scheduling problem of photoreconnaissance satellite. We assume there is a fictive task $o$, which begins and ends the selected sequence (with no time and no visibility constraints). We define set $I^{+}=I \bigcup\{o\}$.

Let us define some notations first.

$p_{i} \quad$ : processing time of task $i, i \in I^{+}$;

$w_{i}$ : the weight of candidate imaging task $i$;

$s_{i, j} \quad$ : setup time from processing task $i$ to $j, i, j \in I^{+}$; 
In this article, three sets of decision variables are used: $X \quad$ : the set of order in selected sequence. If the task $i$ is

followed by task $j$ in the selected sequence, $x_{i, j}=1$, otherwise $x_{i, j}=0$;

$T$ : the set of imaging starting time of tasks, where $T=\left\{t_{0}, t_{1}, \cdots, t_{M}\right\}, M$ is the amount of task in set

$I^{+}$. For any candidate task $i \in I$, if $i$ is selected, $t_{i}$ is the imaging starting time of task $i$, otherwise $t_{i}=0$. There is $t_{0}=0$;

$Y \quad$ : the set of using state of time window. If the task $i$ utilize time window $T W_{n}^{i}, y_{n}^{i}=1$, otherwise $y_{n}^{i}=0, n \in\left\{1, \cdots, N_{i}\right\}$.

The problem can be formulated as follows:

$$
\begin{aligned}
& \max \sum_{i \in I^{+}} w_{i}\left(\sum_{j \in\left(I^{+}-i\right)} x_{i, j}\right) \\
& \text { s.t. } \quad \sum_{j \in\left(I^{+}-i\right)} x_{i, j} \leq 1 \quad \forall i \in I \\
& \sum_{i \in I} x_{0, i}=1 \\
& \sum_{j \in\left(I^{+}-i\right)} x_{i, j}-\sum_{j \in\left(I^{+}-i\right)} x_{j, i}=0 \quad \forall i \in I^{+} \\
& \sum_{j \in\left(I^{+}-i\right)} x_{i, j}-\sum_{n=1}^{N_{i}} y_{n}^{i}=0 \quad \forall i \in I^{+} \\
& \sum_{n=1}^{N_{i}} y_{n}^{i} w s_{n}^{i} \leq t_{i} \quad \forall i \in I \\
& \sum_{n=1}^{N_{i}} y_{n}^{i}\left(w e_{n}^{i}-p_{i}\right) \geq t_{i} \quad \forall i \in I \\
& \left(t_{i}+p_{i}\right) \sum_{j \in\left(I^{+}-i\right)} x_{i, j}+\sum_{j \in\left(I^{+}-i\right)} s_{i, j} x_{i, j} \leq \sum_{j \in\left(I^{+}-i\right)} t_{j} x_{i, j} \\
& x_{i, j} \in\{0,1\} \quad i, j \in I^{+} \\
& y_{n}^{i} \in\{0,1\} \quad i \in I^{+}, n \in\left\{1, \cdots, N_{i}\right\}
\end{aligned}
$$

The objective (1) of the mathematical model considers the weight sum of selected tasks, which ensures that the selected sequence will achieve the maximum efficiency of satellite. Constraint (2) expresses that an imaging task can be selected once at most. Constraint (3) forces that the fictive imaging task $o$ must be selected. Constraint (4) sates that if an imaging task is selected, the amount of images preceding it must be equal to that of images following it. If an imaging task is not selected, it has no preceding and no following selected image. Constraint (5) states that if an imaging task is selected, it must occupy a time window. If an imaging task $i$ is not selected, none of the time window will be utilized. Constraints (6) and (7) force each selected imaging task must be completed within one time window. Constraints (8) states the time constraint between two selected imaging tasks, while task $i$ is followed by task $j$, the imaging of $i$ should end before the starting of imaging $j$.

The scheduling problem formulated above is a mixed integer programming problem of NP-hard computational complexity. To solve this NP-hard problem, we adopt an advanced tabu search (TS) algorithm.

Tabu search is a method to search the optimum solution for combinatorial optimization problems, which is proposed by Glover $(1989,1990)$. Its power has been shown in solving many hard problems including the maximal satisfiability and graph-coloring problem. TS has three phases: preliminary search, intensification, and diversification. During the first step, TS is similar to some other optimization methods. That is whatever point $x$ in the input space is focused currently, the criterion function $f$ at all neighbors $N$ of $x$ is evaluated to find the new point $x^{\prime}$ even if it is worse than $x$. But this idea creates the possibility of endlessly cycling back and forth between $x$ and $x^{\prime}$; to avoid this TS uses a tabu list of forbidden moves. However, it is difficult to evaluate the length of tabu list while the topological of search space is not clear. To solve the problem of tabu list we adopt a tabu search algorithm with varying neighborhood (VNTS) for the satellite scheduling problem. The neighborhood used can be changed with the process of the algorithm, so that not only the scale of the neighborhood is small, but also the reachability can be kept. The computational results of different scenarios show that the VNTS algorithm has high efficiency and has a more steady performance.

The main procedure of VNTS is shown in Figure 3.

Here $N_{1}$ and $N_{2}$ are the iterative upper bounds. The TSprocess ( ) is a tabu search process, which searches a better solution in neighborhood $H$ with a tabu list of length $L . S_{0}$ is the initial solution of mathematical model. $S^{\prime}$ represents the best solution got within neighborhood $H$.

\subsection{Validating Scheme}

At this stage, simulation module will simulate whole imaging process of photo-reconnaissance satellite based on the scheme, include flying, imaging, and adjusting satellite-based sensor. The results of simulation will be passed back to the scheduling module. If a scheme is found incompatible with 
imaging activities, simulation module will make the scheduling module re-compute until a feasible scheme is produced.

The simulation module fulfils the function of feedback which causes the schedule module amend its scheme to close to feasible solution.

\section{NUMERICAL EXPERIMENTS}

Numerical experimentation is used to verify the feasibility and optimality of the proposed simulation-based scheduling mechanism.

Experiments are done on several cases. Each case includes tens of spot targets and two area targets, those targets are produced randomly by a target producing module. Those experiments are conducted in an Intel $P 41.6 G \mathrm{PC}$ with memory size of $128 \mathrm{MB}$. The time period of the satellite scheduling is 86400 seconds ( 24 hours). Those experiments show that our simulation-based scheduling system could effectively solve the problem of mixed imaging mission which includes both spot targets and area targets.

$$
\begin{aligned}
& \text { VNTS a } \lg \text { orithm } \\
& \text { begin } \\
& \text { bestValue } \leftarrow 0 \\
& S_{0} \leftarrow \text { Initial solution } \\
& S_{k} \leftarrow S_{0} \\
& \text { repeat } \\
& \text { loopParam } \leftarrow 0 \\
& H \leftarrow H_{1} / / \text { set neighbourhood to be } H_{1} \\
& L \leftarrow L_{1} / / \text { set length of tabu list to be } L_{1} \\
& \text { while }(\text { loopParam } \leq N) \text { do } \\
& \text { TSprocess (bestValue, } H, L, S_{0}, S^{\prime} \text { ) } \\
& \text { loopParam } \leftarrow \text { loopParam }+1 \\
& \text { end while } \\
& S_{0} \leftarrow S_{k} \\
& \text { loopParam } \leftarrow 0 \\
& \mathrm{H} \leftarrow \mathrm{H}_{2} / / \text { set neighbourhood to be } \mathrm{H}_{2} \\
& L \leftarrow L_{2} / / \text { set length of tabu list to be } L_{1} \\
& \text { while (loopParam } \leq M) \text { do } \\
& \text { TSprocess (bestValue, } H, L, S_{0}, S^{\prime} \text { ) } \\
& \text { loopParam } \leftarrow \text { loopParam }+1 \\
& \text { end while }
\end{aligned}
$$

Figure 3: The procedure of VNTS

\section{CONCLUSIONS}

In this paper, we present the simulation-based scheduling mechanism for an photo-reconnaissance satellite. The satellite scheduling problem belongs to single-machine scheduling problem with time window constraint. We utilize simulation module to construct scenario, process data, and validate scheme. We formulate the scheduling problem with a MIP mathematical model, and adopt a varying neighborhood Tabu search algorithm to solve the scheduling problem. We also utilize numerical experiments to assess the optimality of the scheduling mechanism.

\section{REFERENCES}

Lamaitre, M., and Verfaillie, G. 2002. Selecting and scheduling observations of agile satellites. Aerospace Science and Technology, 6, 367-381.

Walton, J. 1993. Models for the Management of satellitebased Sensors. Ph.D. Dissertation, Massachusetts Institute of Technology.

He, R. J. 2004. Research on imaging reconnaissance satellite scheduling problem. Ph.D. Dissertation, National University of Defense Technology.

Lamaitre, M., and Verfaillie, G. 2000. How to manage the new generation of agile earth observation satellites. SpaceOps 2000.

Sun, Y. K., and Liu, M. 2001. Tabu search algorithm with varying neighborhood and its application to Job Shop scheduling problem. ACTA ELECTRONICA SINICA, 29, 621-625.

Lin, W. C., and Liao, D. Y. 2004. Daily imaging scheduling of an earth observation satellite. IEEE Transactions on Systems, Man, Cybernetics.

\section{AUTHOR BIOGRAPHIES}

QIMING RUAN is a doctor candidate in the School of Information System and Management at National University of Defense Technology, China. His research interests includes artificial intelligence, integrated application, scheduling, and optimization in the area of military application. He can be reached via email at <qimruan@163. com>.

YUEJIN TAN is a professor in the School of Information System and Management at National University of Defense Technology, China. His research interests includes planning and scheduling, optimization in the area of manufacturing. His email address is <yjtan@nudt. edu. cn>.

RENJIE HE is an instructor in the School of Information System and Management at National University of Defense Technology, China. He obtained his degree of Ph.D at National University of Defense Technology in 2004. His current research interests includes artificial intelligence, 
integrated application, planning and scheduling, optimization in the area of military application. His email address is $<$ he_rjamsn.com>.

YINGWU CHEN is a professor in the School of Information System and Management at National University of Defense Technology, China. His current research interests includes scheduling, optimization, effectiveness evaluation in the area of national defense. His email address is <ywchen@nudt. edu.cn>. 